\title{
A pharmacological modification of pain and epithelial healing in contemporary transepithelial all-surface laser ablation (ASLA)
}

This article was published in the following Dove Press journal:

Clinical Ophthalmology

15 April 2015

Number of times this article has been viewed

\section{Ioannis M Aslanides \\ Vasilis D Selimis \\ Nikolaos V Bessis \\ Panagiotis N Georgoudis}

Emmetropia Mediterranean Eye Institute, Heraklion, Crete, Greece
Correspondence: loannis Aslanides Emmetropia Mediterranean Eye Institute, Plateia Eleftherias 44, Heraklion, Crete 7I20I, Greece

$\mathrm{Tel}+302810226198$

Fax +30 2810343436

Email i.aslanides@emmetropia.gr
Purpose: We report our experience with the use of the matrix regenerating agent (RGTA) $\mathrm{Cacicol}^{\circledR}$ after reverse transepithelial all-surface laser ablation (ASLA)-SCHWIND to assess the safety, efficacy, pain, and epithelial healing.

Methods: Forty eyes of 20 myopic patients were prospectively recruited to a randomized fellow eye study. Patients underwent transepithelial ASLA in both eyes, with one of the eyes randomly assigned to the use of the RGTA Cacicol. Postoperative pain and vision were subjectively assessed with the use of a questionnaire on the operative day, at 24 hours, 48 hours and 72 hours. Epithelial defect area size was measured at 24 hours, 48 hours, and 72 hours. Uncorrected distance visual acuity (UDVA) and corrected distance visual acuity (CDVA) were assessed at 1 month.

Results: Mean UDVA at 1 month was LogMAR 0.028. The epithelial defect area was $10.91 \mathrm{~mm}^{2}$ and $13.28 \mathrm{~mm}^{2}$ at 24 hours and $1.39 \mathrm{~mm}^{2}$ and $1.24 \mathrm{~mm}^{2}$ at 48 hours for treated and nontreated eyes, respectively. Overall, $50 \%$ and $65 \%$ of treated and nontreated eyes healed by 48 hours. There was no statistically significant difference in the subjective vision between the groups, although vision of patients in the RGTA group was reported to be better. Pain scores were better at 24 hours and 48 hours in the RGTA group but with no statistically significant difference.

Conclusion: The use of RGTA Cacicol shows faster epithelial recovery after transepithelial ASLA for myopia. Subjectively reported scores of pain and subjective vision were better in the RGTA group, although the difference was not statistically significant. There seems to be a consensual acceleration of epithelial healing even in eyes that did not receive treatment. There were no adverse events and no incidents of inflammation, delayed healing, or haze.

Keywords: matrix regenerating agent, photorefractive keratectomy, PRK, transepithelial

\section{Introduction}

Surface ablation, in the form of photorefractive keratectomy (PRK), has been used for years as the mainstay of refractive procedures for the treatment of refractive errors. With the advent of laser in situ keratomileusis (LASIK), the number of surgeons performing PRK has decreased, and LASIK has become the procedure of choice. LASIK carries several advantages over PRK, mainly, the comfort and very quick visual rehabilitation. PRK has the drawbacks of pain and discomfort, associated with the presence of an epithelial defect, as well as the risk of haze, associated with the interaction of the denuded epithelium with the ablated corneal stroma in the absence of Bowman's layer.

On the other hand, in PRK, there is no need for the creation of a flap. ${ }^{1}$ This offers a significant advantage over LASIK with regards to the biomechanical strength and stability of the ablated cornea, as it results in a thicker and more stable corneal bed compared with LASIK. 
There has been great interest in pharmacologically modifying post-PRK pain, ${ }^{2,3}$ as well as in promoting the epithelial surface healing. ${ }^{4}$ Recently, the production of a matrix regenerating agent (RGTA) has been used for the treatment of persistent nonhealing epithelial defects.

RGTA acts by playing the role of glucosaminoglycans, more specifically, heparin sulfate. The absence of glycosaminoglycans is associated with degradation of the extracellular matrix, which can result in corneal thinning, nonhealing epithelial defects, and inflammation. The RGTA agents restore the balance of matrix proteins and cytokines that are normally bound to heparan sulphate and ensure equilibrium in the corneal microenvironment. This in turn, prevents degradation of extracellular matrix proteins, and promotes stromal and, subsequently, epithelial healing. RGTA agents have mostly been used in cases of persistent epithelial defects, ${ }^{5}$ and chronic nonhealing corneal ulcers. ${ }^{6,7}$ RGTA agents were used after penetrating keratoplasty to promote epithelial healing, in a study conducted by Julienne et al. ${ }^{8}$ It was demonstrated that full epithelial healing was achieved in a mean of 2.7 days compared with 4.6 days for all other patients undergoing penetrating keratoplasty in the same center. As they seem to have an effect in these cases, we wanted to investigate the safety and efficacy of their use after modified transepithelial all-surface laser ablation (ASLA) using the SCHWIND AMARIS Laser suite (SCHWIND eye-tech-solutions, Kleinostheim, Germany), in terms of pain and speed of epithelial closure.

Our preferred technique for treatment of myopia is transepithelial ASLA (SCHWIND), as it is proven to be safe and efficient. ${ }^{9}$ We wanted to assess the pain, the rate of epithelial healing, safety, and efficacy with the use of an RGTA agent after ASLA (SCHWIND) for treatment of myopia.

\section{Materials and methods}

This was a pilot case-control study of a cohort (20 patients, 40 eyes) of myopic (range: -2.25 to $-9.75 \mathrm{D}$ ) patients undergoing reverse transepithelial ASLA (SCHWIND). Mitomycin-C was used in all cases with stromal ablation depth of $75 \mu \mathrm{m}$ or more (32 eyes). One of the two treated eyes was randomly selected to receive RGTA drops $\left(\mathrm{Cacicol}^{\circledR}\right.$, Thea, Clermont-Ferrand, France) until the epithelial defect has completely closed. Patients were recruited at Emmetropia Mediterranean Eye Institute, which is a refractive surgery institute in Crete, Greece between July 2014 and December 2014. Inclusion criteria were age greater than 18 years and myopic spherical equivalent (SE) refraction stable for greater than 1 year. Exclusion criteria were abnormal or keratoconic topography, the presence of ocular copathology or previous surgery, inflammatory or infectious corneal disease, or relevant systemic dermatologic or connective tissue disorders. Included patients provided informed consent. The research conformed to the tenets of the declaration of Helsinki, and ethical approval was provided by the Emmetropia institutional review board.

\section{Preoperative assessment}

Preoperative assessment included manifest and cycloplegic subjective refraction and Logarithm of the minimum angle of resolution (logMAR) visual acuity. Slit lamp biomicroscopy, measurement of intraocular pressure and dilated fundoscopy were performed. Preoperative scanning took place using corneal topography with an Orbscan IIz (Bausch \& Lomb, Rochester, NY, USA), along with corneal wavefront analysis using a Keratron Scout Topographer (Optikon SpA, Rome, Italy) and total ocular wavefront measurement using a HartmannShack aberrometer (ORK-Wavefront-Analyzer; Schwind, Kleinostheim, Germany). Finally, pachymetric measurements of the cornea, and the epithelium in particular, were performed using the Optovue OCT (Optovue, Fremont, CA, USA).

\section{Surgical technique}

All treatments were performed using the Schwind AMARIS 750 excimer laser platform. This uses a $193 \mathrm{~nm}, 0.54 \mathrm{~mm}$ super Gaussian profile flying spot laser, six-axis eye tracking, and pseudorandom thermal optimization. Preoperative anesthesia was administered, with proparacaine eye drops instilled three times, 15 minutes before surgery. Povidone iodine $5 \%$ solution was instilled prior to application of an upper lid adhesive drape and speculum.

All treatments had aberration-free profiles (aspheric). Excimer laser application was preceded by standardized wet sponge application: a Merocel ${ }^{\circledR}$ sponge (Medtronic Inc., Minneapolis, MN, USA) dipped in balanced salt solution was applied with three slow, painting movements on the corneal surface, to avoid uneven wetting and, thus, uneven ablation. Single-step laser delivery was carried out immediately afterward and the cornea then cooled with $20 \mathrm{~mL}$ chilled balanced salt solution. In 32 eyes, mitomycin-C $0.02 \%$ was immediately applied for 20 seconds using a damp Merocel sponge, then the eyes were copiously irrigated and dried. Then, one drop of topical ketorolac $0.5 \%$ and one drop of ofloxacin $0.3 \%$ were instilled, and a bandage contact lens (Acuvue Oasys; Johnson \& Johnson, Jacksonville, FL, USA) was inserted. After surgery, all eyes received topical 
ofloxacin $0.3 \%$ four times per day (qid) until removal of the contact lens; dexamethasone $0.1 \%$ drops qid, tapered over 12 weeks; and artificial tear drops for 3 months. RGTA eye drops (Cacicol) were used randomly in 20 eyes. The regime used was application of one drop immediately after the procedure, one drop on the night of the operation, and twice a day on postoperative day 1 and postoperative day 2 . All patients were instructed to avoid taking any oral painkillers unless in severe pain. In such cases, they were advised they could take oral paracetamol.

\section{Postoperative assessment}

Patients were assessed at 24 hours, 48 hours, and 72 hours after the surgery. The epithelial defect was measured with the use of the slit lamp calipers, and the total area was calculated. In all cases, the bandage contact lens was removed upon complete closure of the epithelial defect. The size of the epithelial defect was measured on the slit lamp on each day. Postoperative pain and the vision were also subjectively evaluated on the operative and on the first 3 days, by a questionnaire.

\section{Analysis}

All statistical analyses were performed using Microsoft Excel $^{\circledR}$ (Microsoft Corp, Redmond, WA, USA). Postoperative and temporal changes were compared using paired $t$-tests. A $P$-value less than 0.05 was considered statistically significant. Results were reported using standard graphs for refractive surgery outcomes. Mean uncorrected distance visual acuity (UDVA) was used to assess efficacy, and the preoperative-to-postoperative histogram plotted. Safety was evaluated by the pre- to postoperative change in lines of corrected distance visual acuity (CDVA), and the safety index (mean postoperative CDVA/mean preoperative CDVA). Accuracy was determined by comparing the attempted against the achieved change in SE. Stability was reported in terms of comparison of refractive outcome at postoperative time points.

\section{Results}

The study consisted of 40 eyes of 20 patients (eight males, 12 females). Mean preoperative spherical equivalent (SE) refractive error was $-5.19 \mathrm{D}$ (standard deviation [SD] $1.86 \mathrm{D}$ ). Mean age was 24.95 years (SD 4.08, range 19-33 years).

\section{Laser treatment parameters}

Ablation profile planning was carried out using the integrated ORK-CAM software. The mean planned maximum ablation depth was $143.11 \mu \mathrm{m}$ (SD 54.31, range 91.81-192.88 $\mu \mathrm{m})$. This ablation value includes the epithelium ( $55 \mu \mathrm{m}$ centrally), thus the mean stromal ablation depth was $81.59 \mu \mathrm{m}$, ranging from 36.81 to $137.88 \mu \mathrm{m}$. The mean optical zone was $6.5 \mathrm{~mm}$ (SD 0.13, range 6-6.75 $\mathrm{mm}$ ).

There were no perioperative complications, and all recruited patients underwent the planned treatment according to protocol.

\section{Visual acuity, efficacy, and safety}

Mean LogMAR UDVA at 1 month was 0.028 (SD 0.05) for both groups. At 1 month, the RGTA group had mean LogMAR UDVA of 0.037 , and the non-RGTA group had a mean LogMAR UDVA of 0.02 . There was no statistically significant difference between the two groups $(P=0.29)$. No eye lost two or more lines of vision. The mean SE refractive error at 1 month was $0.09 \mathrm{D}$ (SD 0.17, range 0-0.5 D).

\section{Epithelial defect size}

The epithelial defect area was measured on the slit lamp, in $\mathrm{mm}^{2}$ (maximum vertical size multiplied by vertical horizontal size). The epithelial defect area was smaller on postoperative day 1 for the RGTA-treated eyes but was larger than the nontreated group of eyes on day 2 . The difference was not statistically significant on any of the days. All epithelial defects closed by 72 hours.

\section{Pain}

Pain was assessed with a questionnaire on the patient's perception of pain on a scale of $0-4(0=$ no pain, $4=$ severe pain). On the operative day, the average pain score was greater in the RGTA- vs non-RGTA-treated eyes and was 2.56 (confidence interval $=2.1-2.9$ ) vs $2.44(\mathrm{CI}=2.1-2.7$ ), with $P=0.6$. The pain was less on day 1 , with scores of 1.10 $(\mathrm{CI}=0.7-1.5)$ vs $1.30(\mathrm{CI}=0.85-1.75)$, respectively, with $P=0.51$. On day 2 , the scores were $0.10(\mathrm{CI}=-0.04-0.24)$ vs 0.25 (CI $=0.01-0.49)$, respectively, with $P=0.29$. There was no pain in either group on day 3 . There was no statistically significant difference between the groups; however, the average pain score was less in the RGTA-treated eyes.

All the results are summarized in Figures 1-4.

\section{Subjective vision}

The subjective vision was assessed with the use of a questionnaire on the patient's perception of visual improvement, on a scale of $0-4(0=$ poor vision, $4=$ excellent vision $)$. On the operative day, there was no difference between the two groups of eyes, and the average score was 1.33 


\section{A}

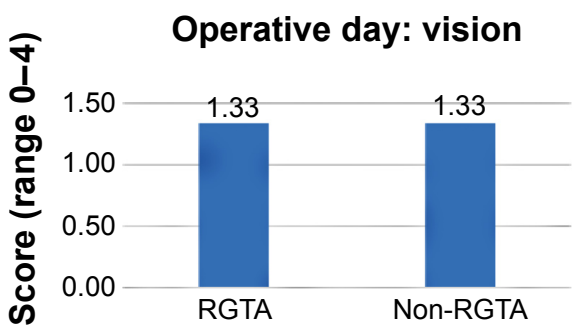

Figure I (A) Vision and (B) pain on the operative day.

Abbreviation: RGTA, matrix regenerating agent.

( $\mathrm{CI}=1.03-1.63)$ in both groups. On all days, the vision was reported to be better in the non-RGTA-treated eyes on day 1 were $1.90(\mathrm{CI}=1.5-2.3)$ vs $1.95(\mathrm{CI}=1.55-2.35)$, respectively, with $P=0.85$. On day 2 the scores were 2.55 ( $\mathrm{CI}=2.2-2.9)$ vs 2.65 ( $\mathrm{CI}=2.35-2.95)$, respectively, with $P=0.66$. On day 3 , the scores were $3.00(\mathrm{CI}=2.7-3.3)$ vs 3.05 (CI $=2.8-3.3$ ), respectively, with $P=0.78$.

\section{Discussion}

In our pilot study, the use of the RGTA agent following transepithelial ASLA seemed to offer a number of advantages compared with the use of the standard regime. The use of the agent itself gave greater amount of comfort to the applied

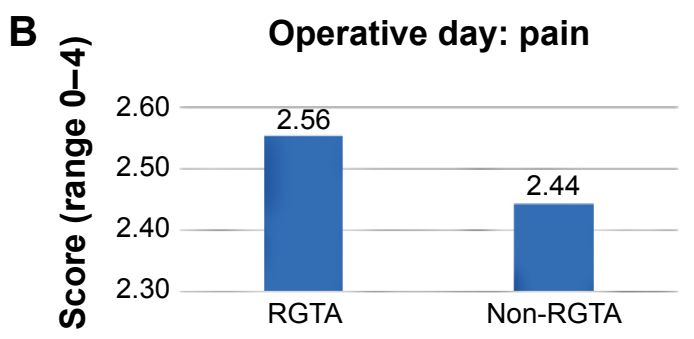

eye, and the pain score in the treated eyes was consistently less than in the nontreated eyes, even though there was no statistically significant difference between the eyes. In eyes that were treated with the RGTA agent, regardless of the size of the epithelial defect, there was less discomfort compared with the nontreated eye. This may be due to the formation of a thin "film" of RGTA, preventing the pain caused by exposed corneal nerves.

The speed of epithelial closure (epithelial defect size) was comparable in both groups. What is very interesting is that $50 \%$ of eyes in the RGTA group and $65 \%$ of eyes in the non-RGTA group demonstrated complete epithelial closure by 48 hours. Moreover, by 48 hours, $70 \%$ eyes in the RGTA
A

$24 \mathrm{~h}$ : vision

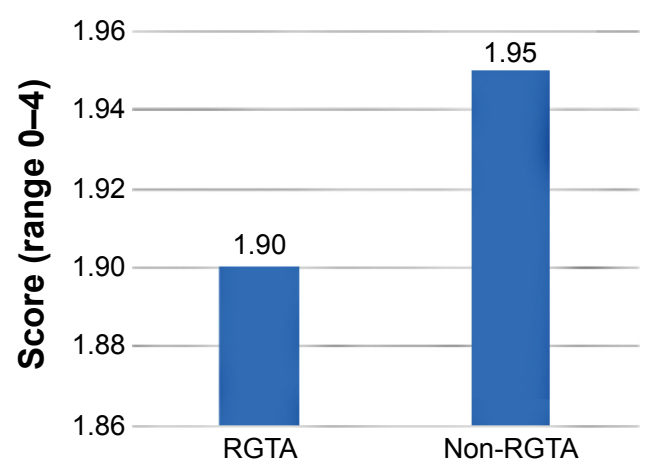

B

$24 \mathrm{~h}$ : pain

1.40

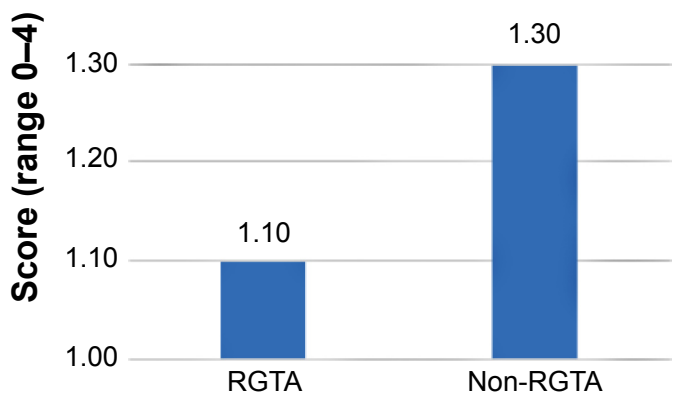

C

$24 \mathrm{~h}$ : epithelial defect

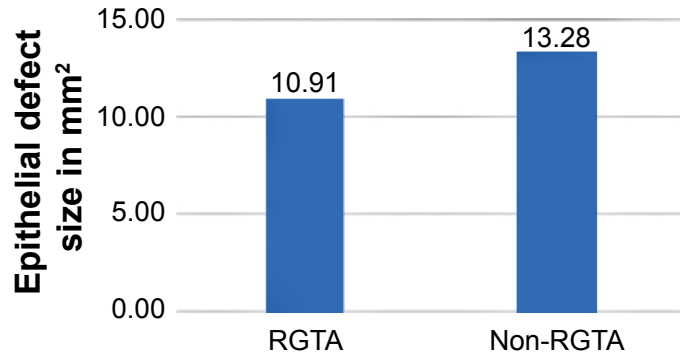

Figure 2 (A) Subjective vision, (B) pain, and (C) size of epithelial defect $\left(\mathrm{mm}^{2}\right)$, at 24 hours. Abbreviation: RGTA, matrix regenerating agent. 

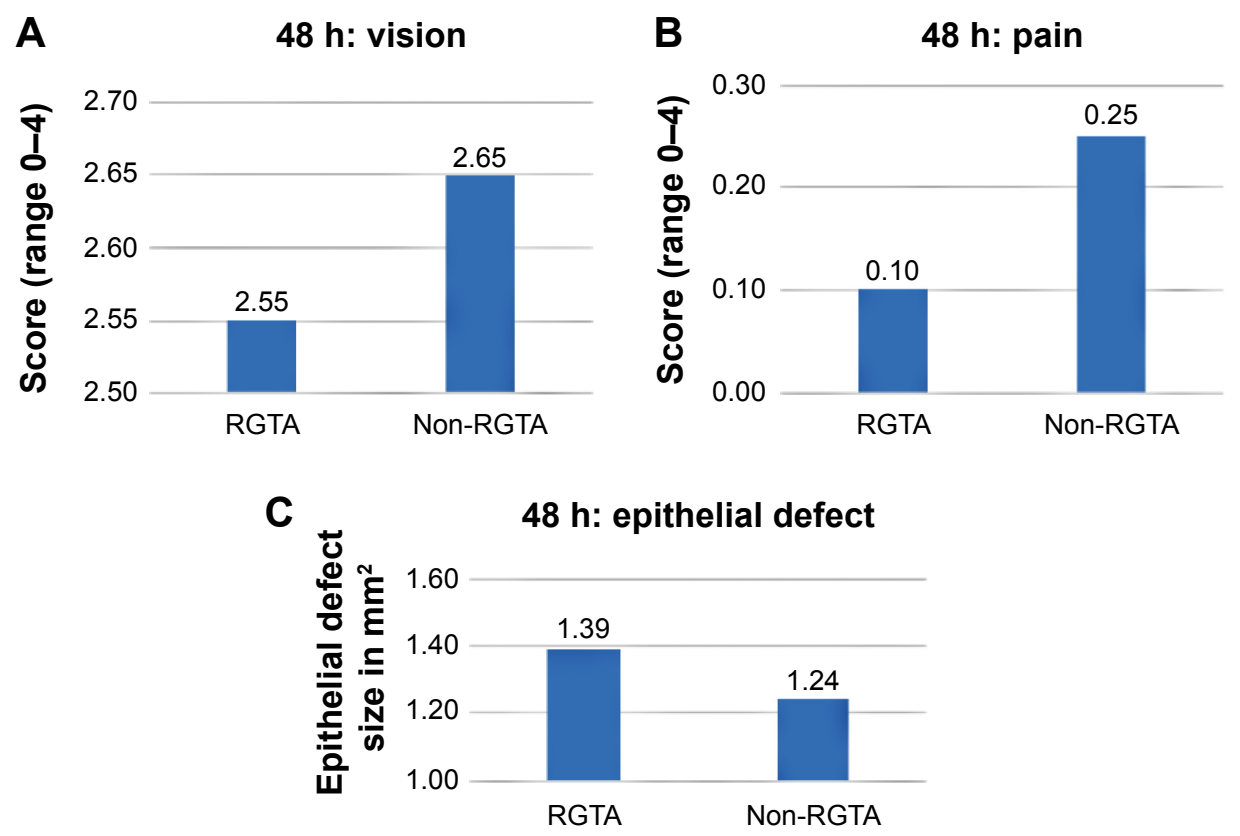

Figure 3 (A) Subjective vision, (B) pain, and (C) size of epithelial defect $\left(\mathrm{mm}^{2}\right)$, at 48 hours.

Abbreviation: RGTA, matrix regenerating agent.

group and $75 \%$ eyes in the non-RGTA group had an area of epithelial defect less than $0.5 \mathrm{~mm}^{2}$. All the eyes show epithelial closure by 72 hours. From our previous experience, after the transepithelial ASLA technique (with the Schwind device), ${ }^{10}$ most eyes (97\%) require at least 72 hours for complete epithelial closure. Our observations suggests that the use of the RGTA exerts an accelerated healing effect in the treated eye, with a consensual effect in the nontreated eye, similar to the one seen glaucomatous patients, where unilateral use of antiglaucoma treatment was shown to decrease intraocular pressure even in the nontreated eye. ${ }^{11}$

The use of the RGTA is safe, and there were no incidence of toxicity, haze, infection, or other adverse event in our study. It is worth mentioning that in some cases, the presence of minute "flour-like" deposits were noted in the eye where

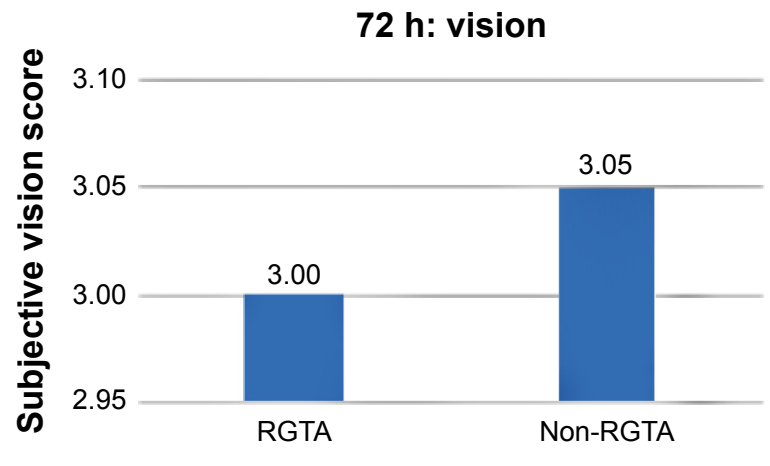

Figure 4 Subjective vision at 72 hours. Abbreviation: RGTA, matrix regenerating agent. the RGTA agent was used; however, there was no impact on the final visual outcome in these patients.

The combination of transepithelial ASLA, offering greater biomechanical stability of the surface ablation procedure, with the RGTA agent, offering greater comfort, makes the use of surface ablations versus LASIK much more appealing, both to the surgeon and the patient.

From the patient experience perspective, the reduced discomfort, which was mostly described as a foreign body sensation rather than as frank pain in the majority of patients, makes the surface ablation much more tolerable.

Another benefit of the use of RGTA is the prevention of postoperative inflammation, which can prevent the cascade of inflammatory events that are also associated with haze. A study conducted by Cejkova et al of alkali-induced chemical injury in rabbits, reported that RGTA facilitated the healing of injured corneas by reducing the proteolytic, oxidative, and nitrosative damage. ${ }^{12}$ The faster recovery of the epithelium possibly reduces the stimulus for migration of keratocytes at the epithelium/stroma interface, which in turn, can reduce the incidence of haze after a surface ablation.

The use of RGTA after surface ablative procedures for correction of refractive errors can significantly reduce the discomfort, which is an inhibiting factor in the decision of the patient to choose a surface ablation over LASIK. It can also offer faster epithelial recovery and contributes to reduced postoperative inflammation, which is very likely to lead to reduced incidence of haze in the long term. 
The combination of a surface ablation with RGTA can reduce the need for LASIK, offering at the same time, greater benefits in terms of reduce risk of postoperative ectasia. It can also contribute to reduced cost of the refractive procedure when the creation of a LASIK flap can be avoided.

\section{Conclusion}

In our pilot study, we report the effect of RGTA Cacicol in the epithelial healing after transepithelial ASLA surface ablation for the correction of myopia. Our patients reported reduction of discomfort in the eyes treated with RGTA. The use of RGTA seemed to promote accelerated epithelial healing compared with both to transepithelial ASLA and conventional PRK, even when the RGTA was used in only one eye, suggesting a consensual effect. The main limitation of the study was the small number of recruited patients. Further studies and greater numbers are required to establish whether the differences become statistically significant. Also, the long-term outcomes need to be assessed. Furthermore, the pain assessment was subjective, as there was no method/ device to objectively measure the pain. These first results are very encouraging and can potentially lead to a shift in direction, toward less invasive procedures that can offer great safety and even less discomfort compared with the traditional surface ablation procedures.

\section{Disclosure}

The authors report no conflicts of interest in this work.

\section{References}

1. Reinstein DZ, Archer TJ, Randleman JB. Mathematical model to compare the relative tensile strength of the cornea after PRK, LASIK, and small incision lenticule extraction. $J$ Refract Surg. 2013;29(7): $454-460$.
2. Verma S, Corbett MC, Marshall J. A prospective, randomized, doublemasked trial to evaluate the role of topical anesthetics in controlling pain after photorefractive keratectomy. Ophthalmology. 1995; 102(12):1918-1924.

3. Woreta FA, Gupta A, Hochstetler B, Bower KS. Management of post-photorefractive keratectomy pain. Surv Ophthalmol. 2013; 58(6):529-535.

4. Baldwin HC, Marshall J. Growth factors in corneal wound healing following refractive surgery: A review. Acta Ophthalmol Scand. 2002; 80(3):238-247.

5. Kymionis GD, Liakopoulos DA, Grentzelos MA, et al. Combined topical application of a regenerative agent with a bandage contact lens for the treatment of persistent epithelial defects. Cornea. 2014; 33(8):868-872.

6. Pison A, Hay A, Dethorey G, Brezin A, Bourges JL. Healing of a resistant corneal neurotrophic ulcer using a new matrix therapy agent (RGTA). Acta Ophthalmologica. 2013;91 Suppl S252:S0.

7. Cochener B, Derrien S. New medical device for chronic corneal ulcers healing. Invest Ophthalmol Vis Sci. 2013;54:3904. Abstract.

8. Julienne R, Haouas M, Trone MC, et al. Topical treatment with a new matrix therapy agent (RGTA, CACICOL-20) improves epithelial wound healing after penetrating keratoplasty. Acta Ophthalmologica. 2014;92 Suppl S253:S0.

9. Aslanides IM, Georgoudis PN, Selimis VD, Mukherjee AN. Single-step transepithelial ASLA (SCHWIND) with mitomycin-C for the correction of high myopia: long term follow-up. Clin Ophthalmol. 2015; 9:33-41.

10. Aslanides IM, Padroni S, Arba Mosquera S, Ioannides A, Mukherjee A. Comparison of single-step reverse transepithelial all-surface laser ablation (ASLA) to alcohol-assisted photorefractive keratectomy. Clin Ophthalmol. 2012;6:973-980.

11. Gibbens MV. The consensual ophthalmotonic reaction. Br J Ophthalmol. 1988;72(10):746-749.

12. Cejkova J, Olmiere C, Cejka C, Trosan P, Holan V. The healing of alkali-injured cornea is stimulated by a novel matrix regenerating agent (RGTA, CACICOL20): a biopolymer mimicking heparan sulfates reducing proteolytic, oxidative and nitrosative damage. Histol Histopathol. 2014;29(4):457-478.
Clinical Ophthalmology

\section{Publish your work in this journal}

Clinical Ophthalmology is an international, peer-reviewed journal covering all subspecialties within ophthalmology. Key topics include: Optometry; Visual science; Pharmacology and drug therapy in eye diseases; Basic Sciences; Primary and Secondary eye care; Patient Safety and Quality of Care Improvements. This journal is indexed on Submit your manuscript here: http://www.dovepress.com/clinical-ophthalmology-journal

\section{Dovepress}

PubMed Central and CAS, and is the official journal of The Society of Clinical Ophthalmology (SCO). The manuscript management system is completely online and includes a very quick and fair peer-review system, which is all easy to use. Visit http://www.dovepress.com/ testimonials.php to read real quotes from published authors. 